\title{
A CLASSICAL CASE OF PREGNANCY INDUCED GINGIVAL ENLARGEMENT: A CASE REPORT
}

Shailendra S. Chauhan ${ }^{1}$, Amit Pandey², Amrita Raj ${ }^{3}$, Ankita Raj ${ }^{4}$, Saket Nigam ${ }^{5}$

\section{HOW TO CITE THIS ARTICLE:}

Shailendra S. Chauhan, Amit Pandey, Amrita Raj, Ankita Raj, Saket Nigam. "A Classical Case of Pregnancy Induced Gingival Enlargement: A Case Report". Journal of Evolution of Medical and Dental Sciences 2014; Vol. 3, Issue 45, September 18; Page: 11104-11108, DOI: 10.14260/jemds/2014/3449

ABSTRACT: Gingival enlargement is a common clinical problem, sometimes associated with specific conditions. This condition finds a unique place in the literature, because it has been associated with a variety of local and systemic factors. In puberty and pregnancy, gingival enlargement can be due to poor oral hygiene, inadequate nutrition, or systemic variation in hormonal stimulation. This case report presents the clinical, histopathological features as well as the treatment of the inflammatory gingival enlargement in pregnancy, where pregnancy altered the tissue metabolism which disturbed the aesthetics and masticatory function of the patient.

KEYWORDS: Pregnancy,Gingival enlargement, Gingivectomy.

INTRODUCTION: Gingival enlargement is a common clinical entity but finds a unique place in the literature, because it has been associated with a variety of local and systemic factors so differential diagnosis becomes an important aspect for complete management of the lesion. Most of the causative factors lead to an unusual hyperplastic tissue response to chronic inflammation associated with local irritants such as plaque, calculus or bacteria.

Hormonal changes occurring during pregnancy and puberty, however, have long been known to be associated with varying types of gingival enlargement. Hormonal changes can significantly potentiate the effects of local irritants on gingival connective tissue. In all forms of enlargements, good oral hygiene is necessary to minimize the effects of systemic factors; gingivoplasty or gingivectomy may be required, but should be done in combination with prophylaxis and oral hygiene instructions (Kapoor et al, 2010).

Generalized gingival enlargement may vary from mild enlargement of interdental papillae to such severe uniform enlargement that the crowns of the teeth may be covered by hyperplastic tissue (Gungormus et al, 2002). The association between gingival enlargement and pregnancy is widely recognized. Increased gingival inflammation during pregnancy follows the hormonal cycle.

It begins with increasing gonadotropin levels and is maintained throughout 4 to 8 months of pregnancy. An increase in the proportion of Prevotella intermedia in bacterial plaque, rather than an increase in total plaque, is instrumental in the development of gingival inflammation during pregnancy. Increased levels of oestrogen and progesterone during pregnancy also stimulate increased gingival vascular permeability and oedema, respectively (McLeod et al, 2009).

CASE REPORT: A 28-year-old woman reported to the Department of Periodontology, Rama Dental College and Hospital, Kanpur, with the complaint of gum swelling in lower anterior region (Fig.1). Patient also complained of difficulty in chewing and concern for the aesthetics. The patient had not previously used any medication known to provoke gingival enlargement. She was in her fourth month of pregnancy when she noticed this enlargement. 
An intra-oral examination revealed the presence of Grade III enlargement in relation to the mandibular anterior teeth region. (Bokenkamp A et al 1994), with gingival bleeding on probing seen at $90(75 \%)$ of 120 sites probed; average probing depth was of 3-6 $\mathrm{mm}$ with deeper pockets in anterior teeth region. The plaque index was found to be fair on examination (Janice S. Ellis et al 1999).

TREATMENT: After taking the thorough case history full mouth scaling and root planning was performed. Patient was motivated for the maintenance of oral hygiene. The tissue was excised by external bevel gigivectomy. Haemostasis was achieved and patient was put on Tablet amoxicillin 500 mg. three times a daily and Tablet Aceclofenac $100 \mathrm{mg}$ twice a daily for five days. The excised tissue was sent for histopathological examination.

The report showed hyperplastic stratified squamous epithelium with underlying connective tissue stroma showing increased collagen fibers and intense inflammatory cell infiltrate in diffuse pattern (Fig.4-5) These features were suggestive of nonspecific inflammatory gingival enlargement.

DISCUSSION: Gingival changes in pregnancy were described as early as 1898, even before knowledge about hormonal changes was available (Kapoor et al, 2010). Incidence of gingivitis in pregnancy varies from around $50 \%$ to $100 \%$ (Buddiga et al, 2012). The primary role of microbial plaque in initiating gingivitis and periodontal destruction is well established. While examining other contributory factors such as the effects of hormonal flux in pregnancy on periodontal disease status, it is mandatory to isolate the effects of plaque (Tilakaratne et al, 2000).

Gingivitis in pregnancy is caused by bacterial plaque, like in non-pregnant individuals. Pregnancy accentuates the gingival response to plaque. The correlation between gingivitis and the quantity of plaque was greater after parturition than during pregnancy, which suggests that pregnancy induces other factors that aggravate gingival response to local irritants (Kapoor et al, 2010). The patient's recent pregnancy makes pregnancy gingivitis an attractive explanation for the hyperplastic gingiva.

High hormone levels during puberty and pregnancy lead to more pronounced gingivitis and possible marginal enlargement, depending on the level of plaque control prior to puberty or pregnancy. An overgrowth of Prevotella intermedia, together with vascular change and oedema associated with high levels of oestrogen/progesterone is the primary explanation for the gingival inflammation and gingival enlargement (McLeod et al, 2009). There is an increase in gingival inflammation between the $14^{\text {th }}$ and 30th weeks of pregnancy and that this will occur despite a fall in the amount of plaque at the dentogingival junction.

This implicates other factors in addition to plaque accumulation to explain the increased inflammation. It was confirmed that there are marked increase in the plasma levels of oestradiol and progesterone between the 14th and 30th weeks of pregnancy but it was not possible to demonstrate a direct association between these increases and the increase in gingival inflammation (O'Neil, 1979).

These results are consistent with a significant role of oestrogen during primate pregnancy in the regulation of cellular proliferation and differentiation within the gingiva (Reynolds et al, 2004).

However, the mechanism by which these steroids increase gingival inflammation is not known. Interleukin-6 (IL-6), a pleiotropic cytokine produced by many cell types including human gingival fibroblasts (hGF), is secreted in response to inflammatory challenges such as bacterial 
lipopolysaccharide and interleukin-1 (IL-1). Cellular proliferation and the number of cells entering the $S$ phase of the cell cycle are significantly increased in mass cultures of fibroblasts stimulated by oestradiol (Ovadia et al, 2007).

CONCLUSION: The local factors i.e. plaque and calculus are known to be responsible for gingival enlargement during pregnancy. The hormonal factors also play a role in aggravating the enlargement. Therefore, the importance of regular check-up and oral prophylaxis cannot be overlooked.

In the present case, size of the hyperplastic tissue was interfering with the patient's ability to chew, speak and was causing serious esthetic problems; therefore, it was excised completely. Future studies conducted on this aspect would provide us with the better information regarding the etiology of pregnancy induced gingival enlargement.

\section{REFERENCES:}

1. Bokenkamp A, Bohnhorst B, Biere C, Albers N, Offner G, Brodehl J: Nifedipene aggravates cyclosporine A induced gingival hyperplasia. Pediatrics Nephrology, 1994; 8 (2): 181-185.

2. Buddiga V, Ramagoni NK, Snehalatha, Mahantesh: Gingival enlargement-a case series. Annals and Essences of Dentistry, 2012; 4 (1): 73-76.

3. Güngörmüs M, Akgül HM, Yilmaz AB, Dagistanli S, Erciyas K: Generalized Gingival Hyperplasia Occurring during Pregnancy. The Journal of International Medical Research, 2002; 30 (3): 353355.

4. Kapoor A, Malhotra R, Grover V, Saxena D: Pregnancy Associated Gingival Enlargement. Journal of Oral Health \& Community Dentistry, 2010; 4 (2): 48-51.

5. McLeod DE, Stoeckel D, Contreras J, Reyes E: Severe Postpartum Gingival Enlargement. Journal of Periodontology, 2009; 80 (8): 1365-1369.

6. O'Neil TC: Plasma Female Sex-Hormone Levels and Gingivitis in Pregnancy. Journal of Periodontology, 1979; 50 (6): 279-282.

7. Ovadia R, Zirdok R, Diaz-Romero RM: Relationship between pregnancy and periodontal disease. Medicine and Biology, 2007; 14 (1): 10-14.

8. Prevalence of Gingival Overgrowth Induced by Calcium Channel Blockers: A Community-Based Study Dr. Janice S. Ellis , Robin A. Seymour , James G. Steele , Pauline Robertson, Tim J. Butler and J. Mark Thomason Journal of Periodontology January 1999, Vol. 70, No. 1: 63-67.

9. Reynolds MA, Aberdeen GW, Pepe GJ, Sauk JJ, Albrecht ED: Estrogen Suppression Induces Papillary Gingival Overgrowth in Pregnant Baboons. Journal of Periodontology, 2004; 75 (5): 693-701.

10. Tilakaratne A, Soory M, Ranasinghe AW, Corea SM, Ekanayake SL, de Silva M: Periodontal disease status during pregnancy and 3 months post-partum, in a rural population of Sri-Lankan women. Journal of Clinical Periodontology, 2000; 27 (10): 787-792. 


\section{CASE REPORT}

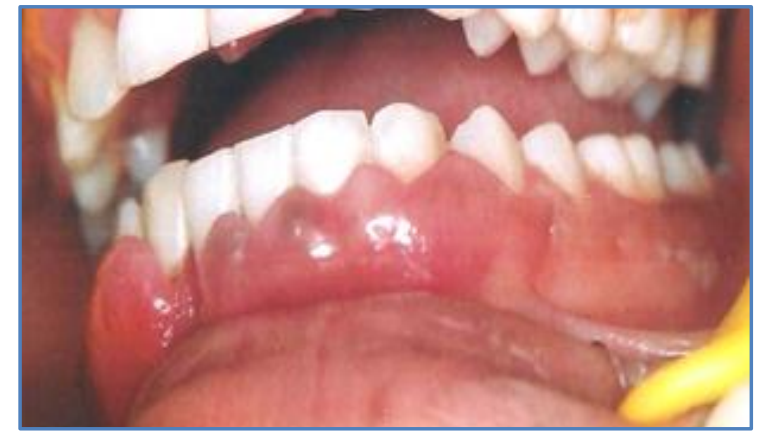

Fig. 1: Photograph showing Pre-operative view

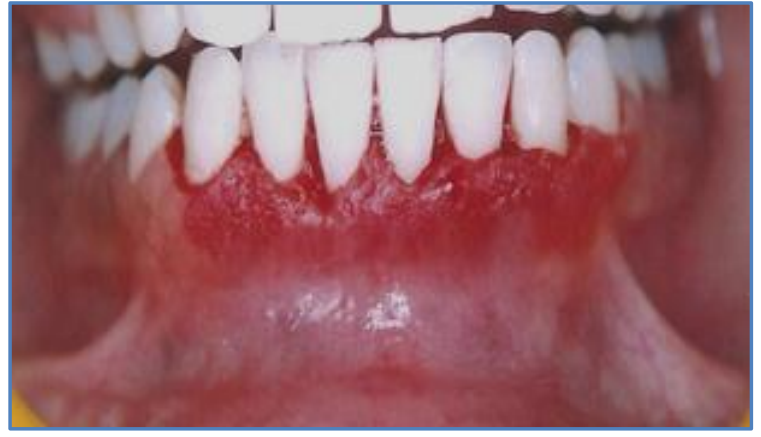

Fig. 2: Photograph showing Post-operative view

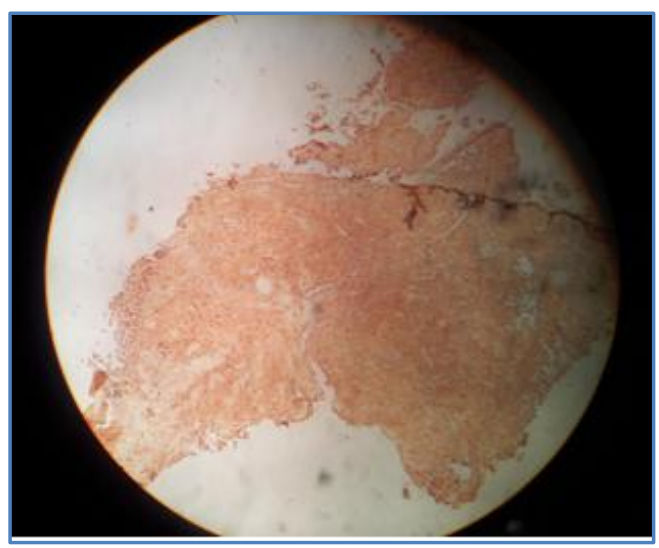

Fig. 3: 4X - scanner

Fig. 4: $10 \mathrm{X}$ - low power view: Showing hyperplastic stratified squamous epithelium with underlying connective tissue stroma showing increased collagen fibers and intense inflammatory cell infiltrate in diffuse pattern.

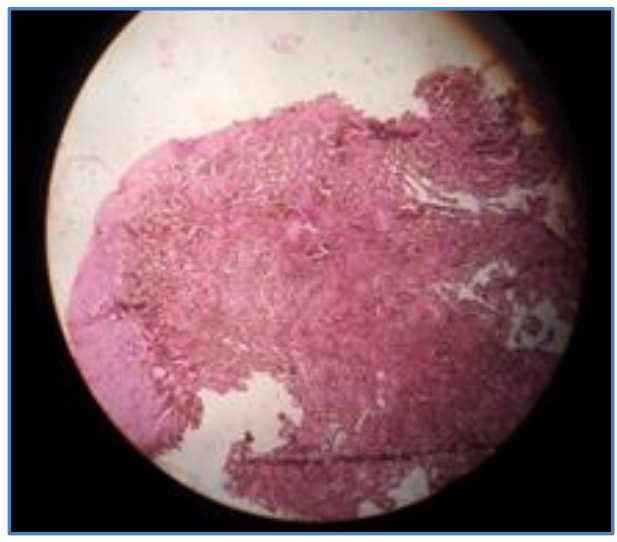

\section{Fig. 4}


Fig. 5: $40 \mathrm{X}$-high power view: Showing hyperplastic stratified squamous epithelium with underlying connective tissue stroma showing increased collagen fibers and intense inflammatory cell infiltrate in diffuse pattern.

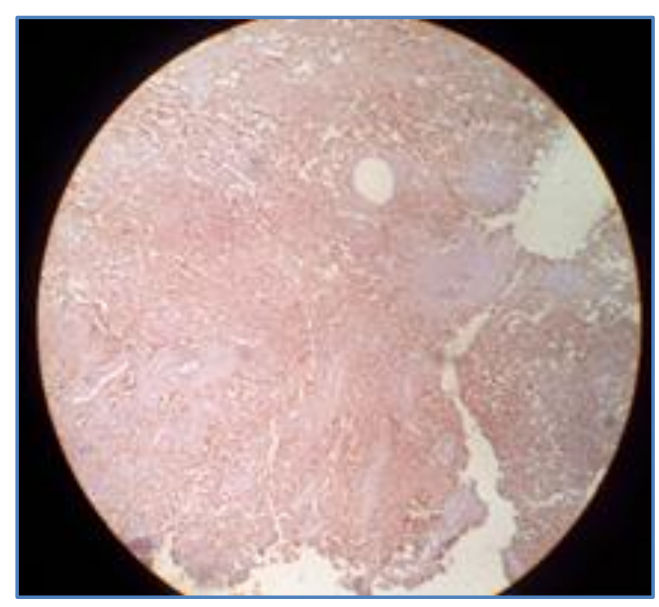

Fig. 5

\section{AUTHORS:}

1. Shailendra S. Chauhan

2. Amit Pandey

3. Amrita Raj

4. Ankita Raj

5. Saket Nigam

\section{PARTICULARS OF CONTRIBUTORS:}

1. Senior Lecturer, Department of Periodontics, Rama Dental College, Kanpur.

2. Senior Lecturer, Department of Periodontics, Rama Dental College, Kanpur.

3. Senior Lecturer, Department of Oral Pathology, Rama Dental College, Kanpur.

4. Senior Lecturer, Department of Oral surgery, Rama Dental College, Kanpur.
5. Assistant Professor, Department of Radiology, Rama Medical College, Kanpur.

\section{NAME ADDRESS EMAIL ID OF THE} CORRESPONDING AUTHOR:

Dr. Amit Pandey, 617, Lakhanpur,

Kanpur.

Email: dramitpandeydental@gmail.com

Date of Submission: 30/08/2014.

Date of Peer Review: 01/09/2014.

Date of Acceptance: 11/09/2014.

Date of Publishing: 18/09/2014. 